\title{
Poster: Manipulation Techniques of 3D Objects Represented as Multi-Viewpoint Images in a 3D Scene
}

\author{
Juan Carlos Yu* \\ Nara Institute of Science and Technology \\ Goshiro Yamamoto ${ }^{\dagger}$ \\ Jun Miyazaki \\ Mark Billinghurst ${ }^{\S}$ \\ HIT Lab NZ \\ Nara Institute of Science and Technology \\ Nara Institute of Science and Technology \\ Hirokazu Katoll \\ Nara Institute of Science and Technology
}

\begin{abstract}
In this poster, we explore manipulation of an object represented by an image-based rendering approach in a 3D scene. We focus on two manipulation techniques that address the problems with using an image-based rendering approach and the constraints imposed by implementing such a system on a mobile device. We present results from our preliminary experiments.
\end{abstract}

Index Terms: H.5.2 [Information Interfaces and Presentation]: User Interfaces-Interaction Styles; I.3.6 [Computer Graphics]: Methodology and Techniques-Interaction Techniques

\section{INTRODUCTION}

Traditionally, scenes are represented as purely 3D graphics (with all entities represented as 3D objects) or through a collection of 2D images. We explore the idea of representing the entire scene as a 3D model while representing a variable object in the scene through an image-based rendering approach. This approach could be useful in object arranging applications similar to My.Ikea [3]

Using an image-based rendering approach to represent an object introduces some constraints, most notably the lack of viewpoints which are solved by using techniques such as image-based modeling [2] and view morphing [5]. We also want to deploy our system on a mobile device. Although mobile device computing power and speed have increased immensely recently, it is still an issue when a mobile device is used to implement such a system. In this regard, we explore two manipulation techniques that do not provide users with additional viewpoints of the object, but allow them to rotate the object at any angle while adhering to some natural constraints.

We conducted a preliminary user study to gain better understanding of the proposed techniques and to study the effect of changing the number of images to represent the target object on user performance and preference.

In this poster we use three terms to pertain to different elements: 1. Target object - the variable object represented through an imagebased rendering approach. 2. Target scene - the 3D scene (without the target object). 3. Combined scene - the resulting scene when the target object is placed inside the target scene.

\section{Related literature}

Image-based rendering techniques have been explored in earlier research to represent 3D objects. Patro [4] created a system that will allow the user to model an object in 3D. They used networkconnected iPhones to acquire synchronized images of an object

\footnotetext{
*e-mail: juan-c@is.naist.jp

†e-mail:goshiro@is.naist.jp

†e-mail:miyazaki@is.naist.jp

§e-mail:mark.billinghurst@hitlabnz.org

Ile-mail:kato@is.naist.jp
}

IEEE Symposium on 3D User Interfaces 2012

4-5 March, Orange County, CA, USA

978-1-4673-1205-9/12/\$31.00 @2012 IEEE which were then uploaded to a server for 3D reconstruction. We envision a system that is fully functional even without external hardware or apparatus. With the constraints imposed by mobile devices, constructing a 3D model from images is unviable.

The image-based rendering technique we used presents the user with an image of the target object generated from a structured collection of images of the target object, as seen through a virtual camera's position in the scene space. This is similar to the research approach used by QuickTime VR [1].

QuickTime VR first requires the user to gather numerous snapshots of the target object at precise angle increments (ideally every 10 degrees). These are then organized in a structured collection to produce an interactive 3D experience for the user. Two problems arise when using the system: 1 . To gather the images, a special apparatus is needed and 2 . To produce a $3 \mathrm{D}$ experience the angle increments should be kept to a minimum.

To preserve the 3D experience with a small image collection, view morphing between each image can produce intermediate views that were previously unavailable [5]. On current mobile devices this approach is not feasible because it is computationally expensive, which may lead to a delay in rendering the output based on user interaction leading to a loss in the overall 3D experience.

The two manipulation techniques that we propose will allow users to rotate the object at any angle by taking advantage of the orientation relationship between the target scene and object.

\section{Proposed Manipulation Techniques}

In general, from a starting orientation (Fig. 1a) when a single object is rotated inside a scene its final orientation relative to the scene is changed (Fig. 1b). To achieve the similar orientation, the scene can be rotated in the opposite direction but in equal magnitude while keeping the object static (Fig. 1c). Both proposed techniques follow this basic concept. Their difference lies in the sequence of actions in which the end viewpoint is achieved.

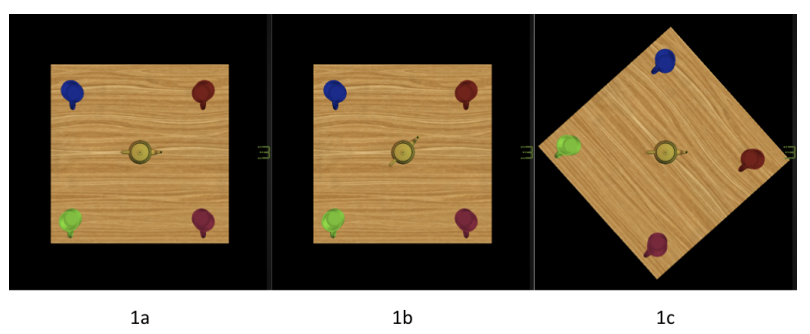

Figure 1: The basic concept behind the two proposed manipulation techniques.

\subsection{Proposed Technique 1 (PT1)}

For PT1, when the user executes a rotation (r) on the target object, the target scene is adjusted to rotate in the opposite direction 
with equal magnitude while the target object is kept static. The scene is continually rotated until the accumulated rotation reaches a threshold ( $T_{a}$ - half the angle increment between each successive object image taken in the Y-axis). The object is then rotated and the scene's orientation, adjusted to preserve their relative orientation.

Table 1: Summary of PT1

\begin{tabular}{ll|ll}
\hline \hline \multicolumn{3}{c}{ if $r>0$} \\
\hline & & Target Object & Target Scene \\
\hline if & $-r>T_{a}$ & currentImage & $-r$ \\
else if & $-r \leq T_{a}$ & nextImage & nextImage.angle $-r$ \\
\hline \multicolumn{4}{c}{ else if $r<0$} \\
\hline if & $-r<T_{a}$ & currentImage & $-r$ \\
else if & $-r \geq T_{a}$ & prevImage & prevImage.angle $-r$ \\
\hline
\end{tabular}

\subsection{Proposed Technique 2 (PT2)}

For PT2, when the user executes a rotation (r) on the target object, the object is rotated together with the target scene to the nearest viewpoint angle $\left(T_{b}\right)$. The scene is continually adjusted until it returns to its initial orientation while the object is kept stationary. After which, the object and the scene are again rotated in a manner that preserves their relative orientation to each other.

Table 2: Summary of PT2

\begin{tabular}{l|ll}
\hline \hline \multicolumn{3}{c}{ if $r>0$} \\
\hline & Target Object & Target Scene \\
\hline if initial rotation & nextImage & $T_{b}-r$ \\
else if $0<T_{b}-r$ & currentImage & $T_{b}-r$ \\
else if $0 \geq T_{b}-r$ & nextImage & $T_{b}+\left(T_{b}-r\right) ; r=0$ \\
\hline \multicolumn{3}{c}{ else if $r<0$} \\
\hline if initial rotation & prevImage & $-T_{b}-r$ \\
else if $0>-T_{b}-r$ & currentImage & $-T_{b}-r$ \\
else if $0 \leq-T_{b}-r$ & prevImage & $-T_{b}+\left(-T_{b}-r\right) ; r=$ \\
& & 0 \\
\hline
\end{tabular}

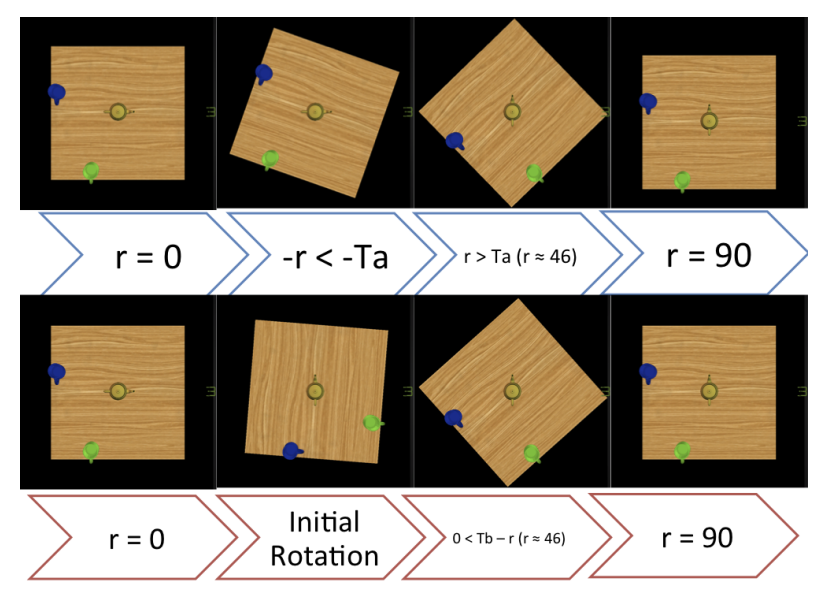

Figure 2: With a positive $\mathbf{r}$ and $T_{a}=45$ and $T_{b}=90$, the displayed output when the user rotates from 0 to 90 degrees.

To illustrate the two manipulation techniques' differences, figure 2 is a sequence of snapshots of the combined scene after a rotation (from 0 deg. to $90 \mathrm{deg}$ ) by the user.

\section{Preliminary User Study}

To evaluate our manipulation techniques, we made an initial prototype using an iPad 2. The ordered image collection representing the target object was gathered through OpenGL. A total of 24 people participated in the user study.

The first experiment focused on the performance of the two techniques. Two tasks were completed using three different manipulation tools/conditions (Table 3). The Conventional tool set refers to manipulation techniques that are common in $3 \mathrm{D}$ user interfaces. The difference between task 1 and 2 was their goal orientation: Task 1 did not require small target scene adjustments while task 2 did. The Conventional tool set and PT2 tool set had no significant difference in terms of performance. For the second task, the Conventional tool set and PT1 had no significant difference in performance. However, the preference data suggest that for both tasks, the users preferred to use the Conventional tool set.

Table 3: The list of available manipulation techniques for each tool set/condition in Experiment 1.

\begin{tabular}{|l|c|c|c|}
\hline \multirow{2}{*}{ Target } & \multicolumn{3}{|c|}{ Conditions: Tool set } \\
\cline { 2 - 4 } & Conventional & PT1 & PT2 \\
\hline Combined Scene & Rotate & Rotate & Rotate \\
\hline Target Scene & Rotate & - & - \\
\hline \multirow{2}{*}{ Target Object } & Translate & Translate & Translate \\
\cline { 2 - 4 } & Rotate & PT1 & PT2 \\
\hline
\end{tabular}

The second experiment explored the effect of increasing the angle increments in between each successive image taken in the Yaxis. The four different angle increments were: 22.5, 30, 45 and 90 degrees respectively. We found that there was no significant difference between 22.5, 30 and 45-degree increments in terms of user preference, but 90 degree increments proved to be different.

The Post Study Interview suggested that the two techniques can be easily used after some practice.

\section{Conclusion ANd Future Work}

Although the participants preferred to use the Conventional tool set, we were made aware that the two techniques can be easily used after some practice. The performance of both techniques was comparable to the conventional tools. Also we found out that we can lessen the number of snapshots making our system more usable.

We will first improve the prototype by modeling a more realistic target scene and addressing issues from the first user study. We will then conduct more user studies using this new prototype. Lastly, we will be making a program that will gather the snapshots of the target object.

\section{REFERENCES}

[1] S. E. Chen. Quicktime vr - an image-based approach to virtual environment navigation, 1995.

[2] P. E. Debevec, C. J. Taylor, and J. Malik. Modeling and rendering architecture from photographs: a hybrid geometry- and image-based approach. In Proceedings of the 23rd annual conference on Computer graphics and interactive techniques, SIGGRAPH '96, pages 11-20, New York, NY, USA, 1996. ACM.

[3] IKEA. My.ikea: Mobile augmented reality application for ikea ps range. http://www.ikeafans.com/home/ mobile-augmented-reality-application-for-ikea-ps-range, Oct. 2009.

[4] R. Patro, C. Y. Ip, S. Bista, and A. Varshney. Social snapshot: A system for temporally coupled social photography. IEEE Computer Graphics and Applications, 31:74-84, 2011.

[5] S. M. Seitz and C. R. Dyer. View morphing. In SIGGRAPH96, pages 21-30, 1996. 